\title{
PROMIS-43 Adult Profile v2.0
}

National Cancer Institute

\section{Source}

National Cancer Institute. PROMIS-43 Adult Profile v2.0. NCI Thesaurus. Code C121683.

A 43-item multidimensional and universal measure of health intended for use across a variety of conditions. It measures an adult patient's self-reported health status in seven domains: physical function, anxiety, depression, fatigue, sleep disturbance, pain interference, and participation in social roles. 